\title{
A novel MOEMS pressure sensor: Modelling and experimental evaluation
}

\author{
PATRICIA M NIEVA*, JIM KUO, SHIUH-HUAH W CHIANG and \\ ABDULLAH SYED
}

Department of Mechanical and Mechatronics Engineering, University of Waterloo, 200 University Avenue West, Waterloo, N2L 3G1

e-mail:pnieva@uwaterloo.ca

\begin{abstract}
This paper presents a novel MOEMS (Micro Opto Electromechanical Systems) pressure sensor suitable for localized precision measurements in high temperature environments. The sensor is based on a micromachined Fabry-Perot device (MFPD) that uses a thin film microcantilever beam as the top mirror and a silicon substrate as the bottom mirror of the optical microcavity. The major effect that the viscosity and density of the air surrounding the MFPD have on the viscous damping provides the mechanism for the detection of the pressure. A major advantage of this configuration is that there is no need for a sealed microcavity since the air is trapped by the viscous damping effects. The sensor has been tested up to $90 \mathrm{psi}$ and pressure sensitivities of about $0.04 \% / \mathrm{psi}$ with a MFPD sensor with a resonant frequency of about $46.7 \mathrm{kHz}$ have been measured.
\end{abstract}

Keywords. MEMS; MOEMS; pressure sensor; harsh environments; Fabry-Perot interferometry; air viscous damping.

\section{Introduction}

Microelectromechanical Systems (MEMS) have emerged as a technology that integrates micromechanical structures with microelectronics, mainly for sensing and actuation applications. A great deal of attention is being drawn to the development of integrated silicon ( $\mathrm{Si}$ )based MEMS to produce smart devices and systems. In automotive or aerospace for example, a misfiring cylinder has a negative impact on the health of the engine and the emissions control. Smart MEMS pressure sensors capable of operating 'in cylinder', where the temperatures are around $400^{\circ} \mathrm{C}$ for automotive engines could continuously monitor the combustion quality in the cylinders reducing emissions and improving fuel economy (Nieva 2007). However, the mechanical and electrical properties of silicon limit the application of Si-based MEMS in harsh environmental conditions. When the environment temperature is too high $\left(>180^{\circ} \mathrm{C}\right)$, conventional microelectronics suffer from severe performance degradation (Goetz 2000). Hence, they must reside in cooler areas or be actively cooled. The additional components such as longer wires, extra packaging and/or bulky expensive cooling systems, add undesired size and weight to the system, which at the same time impact the overall reliability of the system.

*For correspondence 
Remote sensing through optical signal detection has major advantages for safe signal transmission in harsh environments (Eklund \& Shkel 2005). It is highly resistant to electromagnetic interference (EMI) and radio frequency interference (RFI) and at the same time, it eliminates the necessity of on-board electronics, which has been one of the main obstacles in the development of smart Si-based MEMS sensors for high temperature applications. An economical way to deal with higher temperatures and other aggressive environmental conditions is to build MEMS sensors out of robust materials (e.g. Si, silicon nitride, silicon carbide ( $\mathrm{SiC})$ ) and integrate them with optical signal detection techniques to form MOEMS. Fabry-Perot (FP) microstructures have been developed to meet the demand of MEMS sensor systems for harsh environments (Nieva et al 2006; Haueis et al 2001). In this combination, the small and precise size of the sensing elements offer considerable flexibility in choosing the response range and sensitivity of the final sensors. Optical technology has also been used to power a wireless telemetry module for high temperature MEMS sensing and communication (Suster et al 2004). Nevertheless, temperature dependence has been the main weakness of FP microcavities on sensing applications due to the severe degradation of device performance arising from the non-planar deflection of the edge-clamped diaphragm/mirror when it is subjected to external pressure load (Wang et al 2004). In this paper, we present the use of a micromachined Fabry-Perot device (MFPD), previously used as a vibration sensor (Nieva et al 2006), for the development of a new MOEMS pressure sensor for high temperature applications. The major effect that the viscosity and density of the air surrounding the MFPD have on the viscous damping is used as the transduction mechanism for the detection of the pressure. A feature of this mechanism is that both sides of the oscillating mirror are immersed in the pressurized gas and does not require a sealed cavity. Hence, compared to MEMS pressure sensors that are based on sealed cavities and require bonding, the structure is inherently free of mechanical 'creep' due to pressure cycling. In addition, a major advantage of using the MFPD sensor for the measurement of pressure over other FP microcavities is that the temperature dependence of the optical response is minimized by using a cantilevered structure instead of an edge-clamped diaphragm as the top mirror (Wang et al 2004). Analysis and preliminary experimental results are presented to show the sensor's sensitivity and accuracy to measure pressure.

\section{Design and operation}

Figure 1 shows schematically an array of MFPD sensors. Each MFPD consists of a cantilever beam which forms the top mirror of the Fabry-Perot interferometer while the substrate below provides the bottom mirror. These two mirrors form an optical microcavity for a monochromatic laser beam incident at the top. For this cavity arrangement, the total back-reflected interferometric light depends on the height of the optical microcavity at the location where the light beam is directed (spot). When the substrate vibrates, there is a relative deflection of the beam with respect to the substrate $\left(\delta_{r}\right)$ and hence a change in the microcavity height $\left(h_{0}\right)$. If the mechanical characteristics of the device are known, the frequency response of the MFPD sensor can be determined at different pressures by measuring the back-reflected light. Figure 2 shows an SEM picture of an array of Fabry-Perot MOEMS pressure sensors with the top mirror fabricated in LPCVD (low pressure chemical vapour deposition) silicon nitride and designed to operate at a fundamental resonant frequency of $\sim 45 \mathrm{kHz}$. Table 1 lists the main design parameters that were measured for this same array of sensors.

In a previous paper (Nieva et al 2006) it has been shown that at high frequencies and if the air gap is small enough, the air can be confined almost completely under the microbeam 

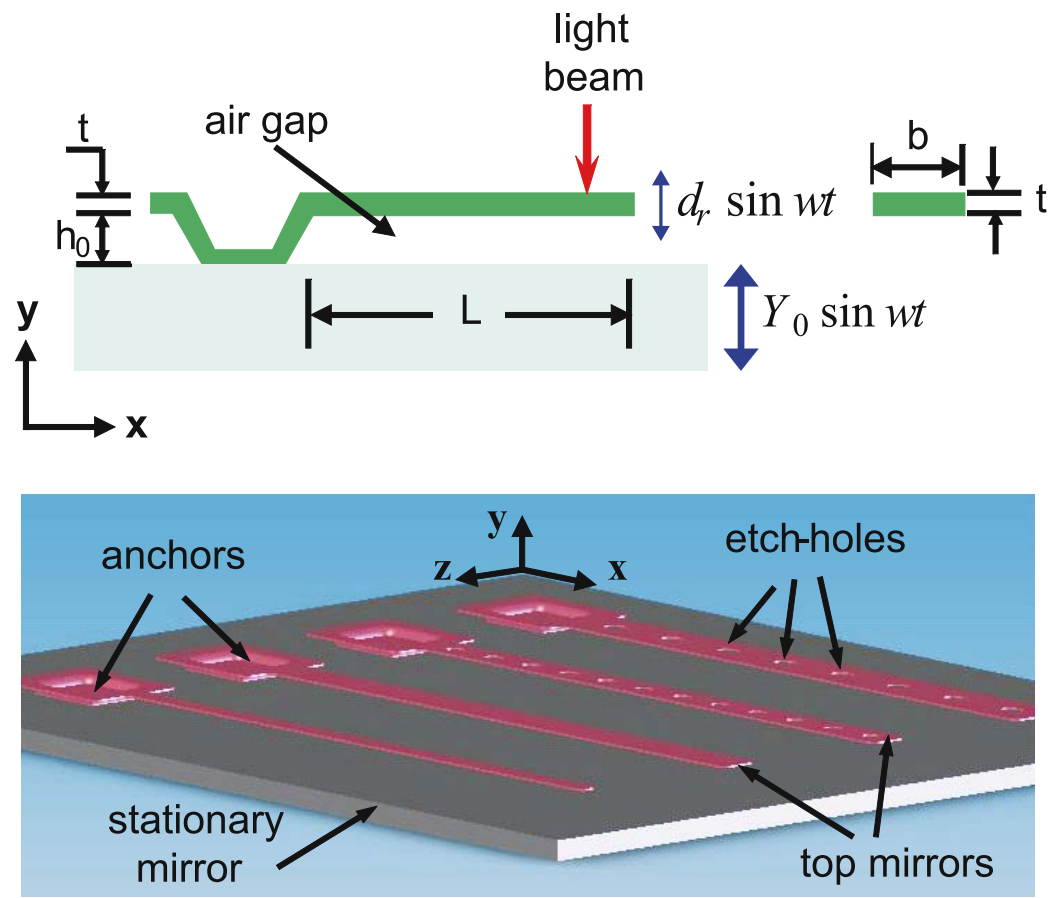

Figure 1. Schematic of the MOEMS Fabry-Perot microcavity pressure sensor.

by the effects of the squeeze film damping (see figure 2). Since the gas cannot escape, the compressive force adds to the stiffness, raising its resonant frequency and increasing the air viscous damping effect. As mentioned before, a major advantage of the MFPD cantilever beam is that both sides of the oscillating mirror are immersed in the pressurized gas and does not need the use of a bonded sealed cavity, as it is the case for example piezoresistive MEMS pressure sensors, thus avoiding mechanical creep in the transducer due to pressure cycling.

The optical microcavity of the MFPD corresponds to a Fabry-Perot in reflectance and its optical response is given by the power reflectance, $R$, of the top of its surface. Assuming no variation in the top mirror thickness $(t)$ or the relaxed cavity height $(h), R$ is only a function of the top layer thickness $\left(l_{1}=t\right)$ and the time-dependant air cavity height $\left(l_{2}=h_{0}+\delta_{r} \sin (\omega t)\right)$ at the location of the laser beam spot and it can be calculated through the transfer matrix method (Nieva et al 2006):

$$
R=|r|^{2}=r r^{*},
$$

where * denotes complex conjugate, and

$$
r=\frac{M_{11} n_{\text {air }}+M_{12} n_{S i} n_{\text {air }}-M_{21}-M_{22} n_{S i}}{M_{11} n_{\text {air }}+M_{12} n_{S i} n_{\text {air }}+M_{21}+M_{22} n_{S i}},
$$

where $n_{\text {air }}$ and $n_{\mathrm{Si}}$ are the index of refraction for air and the silicon substrate, respectively, and $M_{11}, M_{12}, M_{21}$, and $M_{22}$ are the elements of the total transfer matrix $M=M_{1} M_{2}$ given by

$$
M_{i}=\left[\begin{array}{cc}
\cos k_{i} l_{i} & -\frac{j}{n_{i}} \sin k_{i} l_{i} \\
-n_{i} \sin k_{i} l_{i} & \cos k_{i} l_{i}
\end{array}\right]
$$




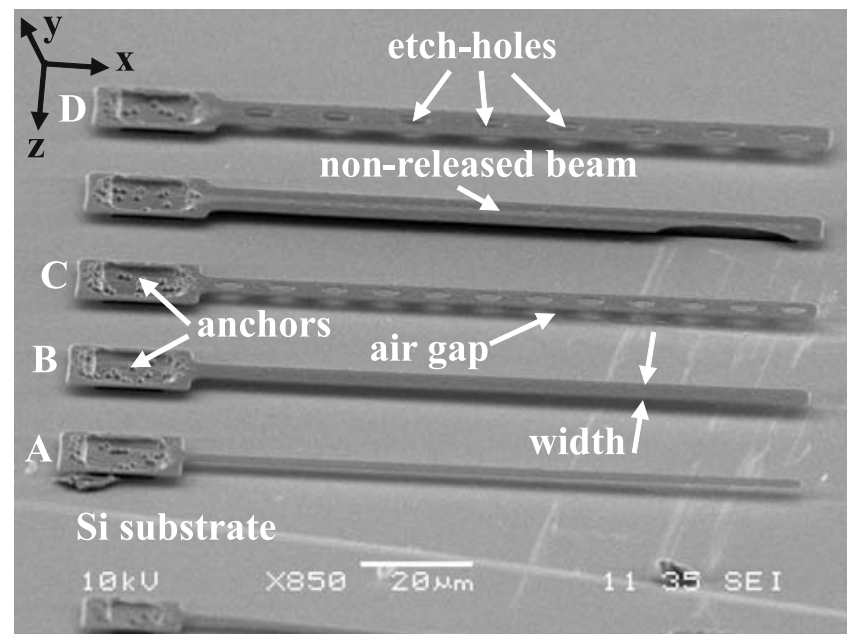

Figure 2. Fabry-Perot MOEMS pressure sensors with the top mirror fabricated in LPCVD silicon nitride and designed to operate at a fundamental resonant frequency of $\sim 45 \mathrm{kHz}$ (Nieva et al 2006).

Table 1. Summary of measured parameters for the Fabry-Perot MOEMS, pressure sensors shown in figure 2 (Nieva et al 2006).

\begin{tabular}{lccccc}
\hline $\begin{array}{l}\text { Beam } \\
\text { type }\end{array}$ & $\begin{array}{c}\text { Length } \\
(\mu \mathrm{m})\end{array}$ & $\begin{array}{c}\text { Width } \\
(\mu \mathrm{m})\end{array}$ & $\begin{array}{c}\text { Etch-hole } \\
\text { diameter }(\mu \mathrm{m})\end{array}$ & $\begin{array}{c}\text { Air gap @ spot } \\
(\mu \mathrm{m})\end{array}$ & $\begin{array}{c}\text { Spot location } \\
(\mu \mathrm{m})\end{array}$ \\
\hline A & 122.5 & 4.25 & - & 2.84 & 5 \\
B & 119.6 & 8.17 & - & 2.66 & 17.5 \\
C & 123.5 & 9.31 & 3.92 & 2.60 & 17.5 \\
D & 118.5 & 13.39 & 5.56 & 2.62 & 22.5 \\
\hline
\end{tabular}

where $k_{i}=\frac{2 \pi}{\lambda} n_{i}$ and $l_{i}$ are the propagation constant and thickness of the $i$ th mode respectively.

Figure 3 shows the typical power reflectance measured for a MFPD sensor as a function of the relaxed air gap height (dotted curve). This function was obtained for the MFPD type A listed in table 1 and shown in figure 2 which has an upper mirror thickness of around $0.45 \mu \mathrm{m}$.

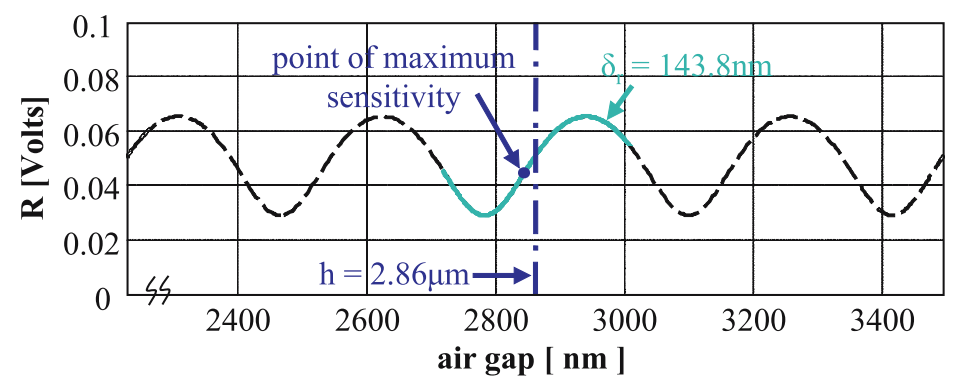

Figure 3. Reflectance of the MFPD type A shown in figure 2 as a function of air gap height (Nieva et al 2006). 


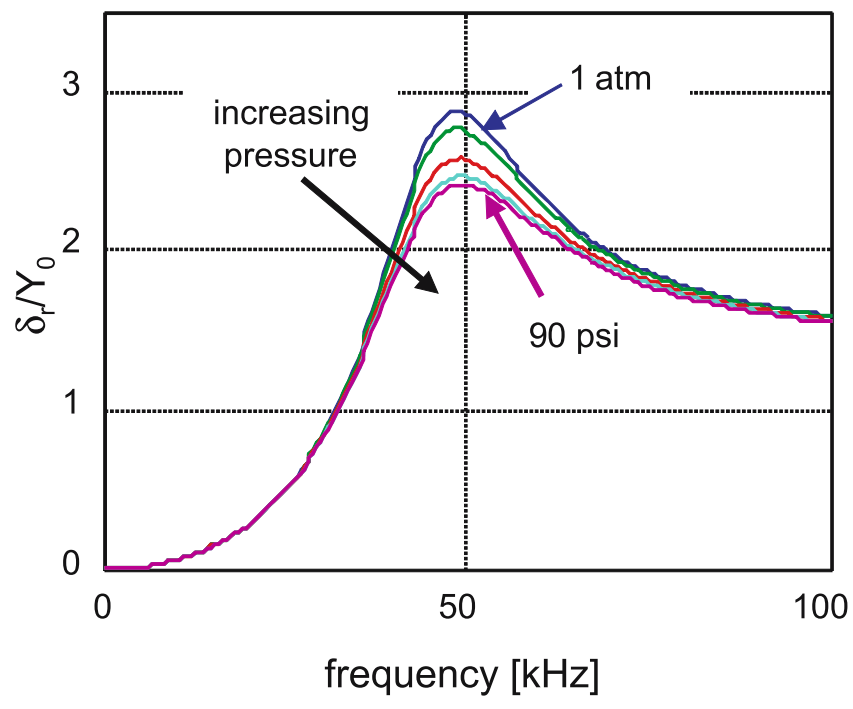

Figure 4. Calculated frequency response of the MFPD type $B$ is shown in figure 2 (listed in table 1 ) as a function of pressure varying between $1 \mathrm{~atm}$ and 90 psi.

The optical signal was measured while the MFPD was at a frequency of $62 \mathrm{kHz}$ and fitted to the AC component of the theoretical power reflectance model described by equations (1) to (3) with $h, \delta_{r}$, and $t$ as the fitting parameters. For the data shown, the relaxed air cavity height, at the location of the light beam spot, was calculated to be about $2.86 \mu \mathrm{m}$. This relaxed cavity height is very close to the point of maximum optical sensitivity which corresponds to $2 \cdot 84 \mu \mathrm{m}$ (see figure 3 ).

\section{Theory}

The pressure-dependent frequency response of the MFPD is calculated using the mechanical theory developed Nieva et al 2007. Figure 4 shows the simulated frequency response for a beam type B, similar to the one listed in table 1 and shown in figure 2 .

The frequency response was calculated for a Young's modulus of silicon nitride of $238 \mathrm{GPa}$ (Nieva et al 2006), an operation temperature of $70^{\circ} \mathrm{C}$, and pressures varying between $1 \mathrm{~atm}$ and 90 psi. The curves shown in figure 4 correspond to pressures of 14.7, 25, 50, 75, and 90 psi. As it can be seen from this figure, a change in pressure causes a shift in resonant frequency and a change in the air viscous damping effect. Figure 4 also shows that better resolutions can be obtained at lower pressures since the shift in air viscous damping decreases as the pressure increases. The pressure-dependent non-dimensional air viscous damping can be calculated using (Nieva et al 2007)

$$
\zeta_{n}=\frac{I_{n}+c_{a}}{2 \rho A \omega_{n}}
$$

where

$I_{n}=\int_{0}^{L} \rho A c_{s}(x) V_{n}^{2}(x) d x:$

$c_{a}=\frac{3}{2} \pi \alpha\left(\mu_{\text {air }}+b \sqrt{\rho_{\text {air }} \mu_{\text {air }} \omega}\right):$ and,

$\omega$
Dissipation due to squeeze film damping

Dissipation due to airflow damping

frequency of operation/ 


$\begin{array}{ll}\omega_{n} & \begin{array}{l}n \text {-natural resonant frequency for a cantilever beam } \\ \text { (Meirovitch 1986); }\end{array} \\ b & \text { width of the beam; } \\ A & \text { cross sectional area of the beam }(A=b t) \\ & \text { mass and air density; } \\ \rho, \rho_{\text {air }} & \text { air viscosity; } \\ \mu_{\text {air }} & \text { experimental constant (Nieva } \text { et al 2007). } \\ \alpha=5 \cdot 5 & \end{array}$

The $n$th normal mode $V_{n}(x)$ satisfies the free vibration differential equation for a cantilever beam and is given in Meirovitch 1986.

\section{Measurement set-up and experimental results}

\subsection{Optical measurement system}

For the measurement of the optical response, a Helium Neon laser $(632.8 \mathrm{~nm})$ is focused down to a $15 \mu \mathrm{m}$ spot at a normal incidence using focusing optics similar to those used in Nieva et al 2006. The light beam is focused on the desired spot of the FP microcavity by adjusting a micro XYZ stage and the reflected light is directed to a photodiode through a 50-50\% beam. The electrical current generated by the photo detector is then amplified and directed to an oscilloscope controlled from a PC via GPIB using Matlab programs. This electrical signal is then processed to determine the relative deflection of the top mirror with respect to the bottom mirror of the MFPD at the frequency of excitation.

Figure 5 shows schematically the pressure-controlled pressure chamber and the optical measurement set-up. The pressure-controlled chamber was recently designed to measure the frequency response of the proposed MFPD pressure sensor. Inside this chamber, the test chip ( $4 \times 4 \mathrm{~mm}$ in size) is surface-bonded to a PL050 PZT piezoshaker from PI Inc. $(5 \times 5 \mathrm{~mm}$ in size), which at the same time is bonded to the base of the chamber using ultra-thin

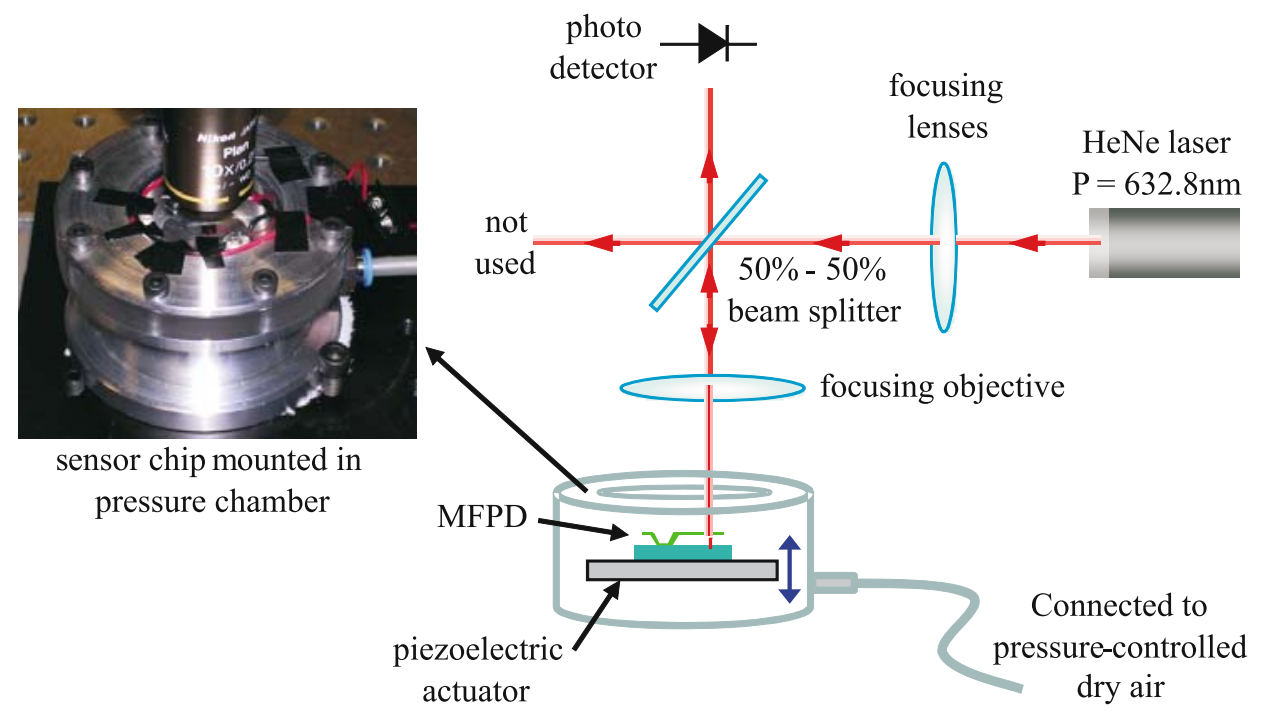

Figure 5. Pressure-controlled chamber and optical measurement set-up for the MFPD pressure sensor. 
double sided tape. A harmonic excitation signal generated by a function generator drives the piezoelectric actuator with a small displacement which is calibrated to approximately $10 \mathrm{~nm}$ amplitude at the surface of the substrate between 2 and $100 \mathrm{kHz}$ using a MSV 300 Polytec Laser Doppler Vibrometer (LDV).

Compared with cumbersome and ambiguous fringe-counting optical detection schemes associated with large cavity FP sensors used in the literature, the convenient FP arrangement of the MFPD sensor has a simpler optical detection scheme. Because the thin film structure and the substrate are approximately parallel, there is no need for optical alignment, wavefront matching, reference arms or sophisticated stabilization techniques. In addition, the simple configuration of the optical interferometric system can be easily integrated in the sensor package.

\subsection{Pressure measurements}

Nieva et al (2007) have shown that the air viscous damping is highly dependent on frequency. Hence, in order to study the operation of the MFPD as a pressure sensor it is important to track its frequency response for a wide range of frequencies including those that are much higher than the resonant frequency of the device. To accurately measure the pressuredependent frequency response of the MFPD sensors, the pressure in the chamber is varied between 14.7 psi and 90 psi. The adjustable air pressure in the chamber is monitored by a MKS pressure gage. The applied pressure changes the air viscous damping and hence the relative displacement of the top mirror with respect to the substrate at different frequencies varying from 2 to $100 \mathrm{kHz}$. This change in relative displacement corresponds to a change in the optical path of the FP microcavity which is sensed by a change in intensity of the light reflected back from the MFPD. The frequency response of the MFPD at different pressures is obtained by plotting the relative displacement of the device as a function of the varying frequency.

Several test structures were tested to evaluate the characteristics of the new MOEMS pressure sensor and the new pressure measurement set-up. Figure 6 shows the non-dimensional

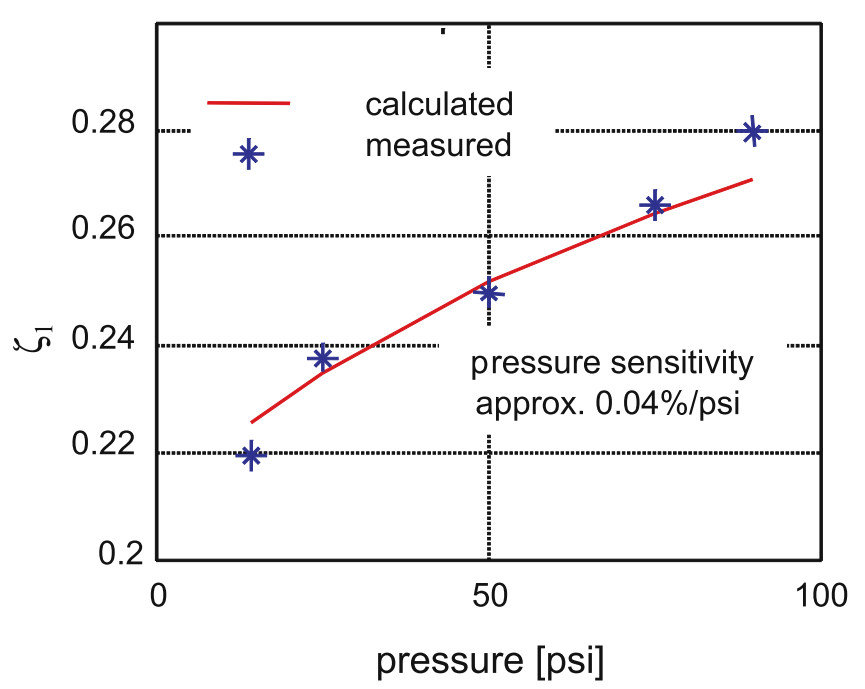

Figure 6. Non-dimensional air viscous damping as a function of applied pressure. Results are shown for analytically calculated and measured values for the MFPD cantilever beam type $\mathrm{B}$ listed in table 1 and shown in figure 1. 
air viscous damping measured at different pressures $(14 \cdot 7,25,50,75$ and $90 \mathrm{psi})$ for the MFPD cantilever beam type B listed in table 1 and shown in figure 2. In this figure, the nondimensional air viscous damping of the structure calculated according to equation (4) is also plotted as a function of applied pressure.

To determine the non-dimensional air viscous damping, the measured MFPD frequency response was fitted to the analytical mechanical model developed in (Nieva et al 2006). The fitted response was used to determine the effective air viscous damping at the different pressures and to determine the temperature of operation of the sensors which was analytically determined to be of about $70^{\circ} \mathrm{C}$. We also found that the analytically calculated natural resonant frequency of $45.4 \mathrm{kHz}$ is slightly lower than the measured natural frequency of $46.7 \mathrm{kHz}$. For these preliminary results, the pressure sensitivity was measured to be about $0.04 \% / \mathrm{psi}$ using the best fit straight line (BFSL) method and could be improved by reducing measurement noise sources, such as intensity fluctuations and a temperature controlled environment. We are re-designing the pressure chamber to measure and control the temperature of operation of the sensor accurately and to test the MFPD sensors at lower and higher pressure ranges. Further testing and analysis of noise sources are needed to characterize the sensor resolution, temperature dependence, and minimum detectable pressure.

\section{Conclusions}

A new MOEMS pressure sensor using a Fabry-Perot microcavity was demonstrated. The key novelty of the proposed design is that the MFPD cantilevered structure alleviates problems such as changes in frequency response and optical path length due to thermally induced stress. Mitigation of these issues is critical to the successful realization of MEMS sensors for high temperature applications. The design also reduces the optical signal averaging effect arising from the non-planar deflection of devices such as multilayered bridges or edge-clamped diaphragms. Because the thin film structure and the substrate are approximately parallel, there is no need for optical alignment, wavefront matching, reference arms or sophisticated stabilization techniques.

A pressure controlled pressure chamber was designed to measure the optical response of the new FP cavity-based MEMS pressure sensors. The measurements have shown reasonable agreement with the analysis, demonstrating that the MFPD sensor is promising in its application as a pressure sensor and offers advantages in terms of size, simplicity, and cost. An important feature of this sensor is that the diaphragm is never loaded by a steady gas pressure. Therefore, the device cannot suffer creep when the pressure is cycled. In addition, the sensor needs not to be sealed since its operation is largely controlled by the air viscous damping which at the same time is highly dependent on the viscosity and density of the gas surrounding the sensor. Preliminary measurements have shown that a damping sensitivity of $0.04 \% / \mathrm{psi}$ can be achieved at pressures varying from $1 \mathrm{~atm}$ to 90 psi with a MFPD cantilever beam of natural resonant frequency of about $46.6 \mathrm{kHz}$. However, more testing is needed to determine the MFPD sensor sensitivity to higher and lower pressure ranges.

\section{References}

Eklund E, Shkel A 2005 Factors affecting the performance of micromachined sensors based on FabryPerot interferometry. J. Micromechanics Microengineering 15: 1770-1776

Goetz J 2000 Sensors that can take the heat. Sensors Magazine 20-38 June 2000 
Haueis M, Dual J, Cavalloni C, Gnielka M, Buser R 2001 A fully packaged single crystalline resonant force sensor. J. Micromechanics and Microengineering 11: 514-521

Meirovitch L 1986 Elements of vibration analysis, (New York: McGraw Hill) 2nd. Ed. Chapter 5

Nieva P, McGruer N, Adams G 2006 Design and characterization of a micromachined Fabry-Perot vibration sensor for high-temperature applications. J. Micromechanics and Microengineering 16: 2618-2631

Nieva P M, Adams G G, McGruer N E 2007 Design and modelling of a multifunctional MEMS Fabry-Perot sensor for the simultaneous measurement of displacement, pressure and temperature. In Proc. of Sensors and Smart Structures Technologies for Civil, Mechanical, and Aerospace Systems, (San Diego: CA, USA) Vol. 6529, pp. 23

Suster M, Ko W, Young D 2004 An optically powered wireless telemetry module for high-temperature MEMS sensing and communication. J. Microelectromechanical Systems 13(3): 536-541

Turner R C, Fuierer P A, Newnham R E, Shrout T R 1994 Materials for high temperature acoustic and vibration sensors: A review. Applied Acoustics 41: 299-324

Wang W, Lin R, Guo D, Sun T 2004 Development of a novel Fabry-Perot pressure microsensor. Sensors and Actuators A116: 59-65

Xiao-qi N, Ming W, Xu-xing C, Yi-xian G, Hua R 2006 An optical fibre MEMS pressure sensor using dual-wavelength interrogation. Meas. Sci. Technol. 17: 2401-2404 